\title{
Ampliación de la distribución de Lutzomyia longipalpis (Lutz \& Neiva, 1912) (Diptera: Psychodidae) en el departamento de Caldas: potencial aumento del riesgo de leishmaniasis visceral
}

\author{
Luz Adriana Acosta ${ }^{1}$, Karina Mondragón-Shem¹, Daniela Vergara ${ }^{1,2}$, Andrés Vélez-Mira1, \\ Horacio Cadena ${ }^{1}$, Lina Carrillo-Bonilla ${ }^{1,3}$ \\ 1 Programa de Estudio y Control de Enfermedades Tropicales, PECET, Universidad de Antioquia, Medellín, \\ Colombia \\ 2 Department of Biology, Indiana University, Bloomington, IN, USA \\ ${ }^{3}$ Facultad de Ciencias Agrarias, Universidad de Antioquia, Medellín, Colombia
}

Introducción. Diversos estudios a nivel taxonómico y molecular sugieren que Lutzomyia longipalpis, principal vector de la leishmaniasis visceral en Latinoamérica, es un complejo de especies con amplia distribución a lo largo del continente y con adaptaciones a diferentes hábitats.

Objetivo. Realizar vigilancia entomológica en el área de influencia de la hidroeléctrica Miel I.

Materiales y métodos. Se utilizaron trampas adhesivas en una intersección de $400 \mathrm{~km}$, aproximadamente, para la captura de insectos, los cuales fueron identificados con la clave taxonómica de Young y Duncan.

Resultados. En este estudio se reporta la presencia de Lu. longipalpis en los municipios de Norcasia a una altitud de $392 \mathrm{msnm}$ y en Marquetalia a $1.387 \mathrm{msnm}$; es el primer reporte a esta altura para esta especie en Colombia.

Conclusiones. Se sugiere que la ampliación de la distribución geográfica y altitudinal de Lu. Iongipalpis en el país podría estar relacionada con la existencia del complejo de especies Lu. Iongipalpis, o a factores ambientales como el aumento de temperatura debido al cambio climático global que viene sucediendo en las últimas décadas. Esta última hipótesis sugeriría que se están creando nuevos hábitats propicios para el establecimiento de Lu. longipalpis, lo cual genera un nuevo riesgo epidemiológico de posibles nuevos focos de leishmaniasis visceral en el país.

Palabras clave: leishmaniasis, Lutzomyia, calentamiento global, zona de riesgo, vigilancia epidemiológica.

doi: http://dx.doi.org/10.7705/biomedica.v33i2.762

Expansion of the distribution of Lutzomyia longipalpis (Lutz \& Neiva, 1912) (Diptera: Psychodidae) in the department of Caldas: Increased risk of visceral leishmaniasis

Introduction: Diverse taxonomical and molecular studies suggest that Lutzomyia longipalpis, main vector of visceral leishmaniasis in Latin America, is a complex of species widely distributed throughout the continent and adapted to different habitats.

Objective: To carry out entomological surveillance in the area of influence of the Miel I Hydroelectric Plant.

Materials and methods: Adhesive traps were used in a transect covering an area of approximately $400 \mathrm{~km}$ for the capture of insects, which were then identified using the Young and Duncan taxonomical key.

Results: This study reports the presence of Lutzomyia longipalpis in the municipalities of Norcasia at an altitude of 392 masl, and in Marquetalia at 1,387 masl.

Conclusions: We suggest that the increase in the geographical and altitudinal distribution could be related to the existence of the Lu. longipalpis species complex, or to environmental factors, such as the rise in temperature due to global climate changes, which create the establishment of Lu. longipalpis, generating a new epidemiological risk for new visceral leishmaniasis foci in the country.

Key words: leishmaniasis, Lutzomyia, global warming, risk zone, epidemiological surveillance.

doi: http://dx.doi.org/10.7705/biomedica.v33i2.762

\section{Contribución de los autores:}

Luz Adriana Acosta, Karina Mondragón-Shem y Lina Carrillo: análisis de resultados.

Daniela Vergara: identificación entomológica.

Lina Carrillo: diseño del estudio.

Todos los autores participaron en el trabajo de campo y elaboración del manuscrito. 
La leishmaniasis visceral es una enfermedad de gran impacto en la salud pública por ser responsable de más de 50.000 muertes cada año y presentar una morbilidad de 500.000 casos anuales a nivel mundial (1). Hasta hace poco era considerada una enfermedad que ocurría principalmente en áreas rurales. Sin embargo, este panorama ha cambiado debido a la presencia de casos en sujetos humanos en áreas urbanas de las grandes ciudades del Viejo Mundo y del Nuevo Mundo (2-5). Una razón del aumento de su distribución pueden ser los cambios que están asociados a problemas socioeconómicos, como el desplazamiento, que aumentan la migración de personas enfermas o reservorios desde las áreas rurales endémicas a las urbanas, en donde junto con la presencia o aparición simultánea del vector, la enfermedad puede propagarse fácilmente. La aparición del vector en estas nuevas áreas urbanas puede deberse a cambios en el ambiente, como factores climáticos o ecológicos que facilitan su colonización (5-8).

Con presencia sólo en América, Lutzomyia longipalpis (Lutz y Neiva, 1912) es una especie flebotomínea de gran importancia, ya que se considera el vector principal de Leishmania infantum (Nicolle, 1908), agente etiológico de la leishmaniasis visceral (9-11). Esta especie presenta adaptaciones a diferentes sistemas ecológicos y se encuentra distribuida en zonas tropicales en una variedad de hábitats, desde áreas rocosas, áridas y semiáridas, hasta muy húmedas y boscosas (12-14).

Existe mucha información para afirmar que $L u$. longipalpis es un complejo de especies (15-19), debido a su amplia y fragmentada distribución geográfica discontinua que va desde el sur de México hasta el norte de Argentina $(12,20)$, incluyendo todos los países de Centroamérica, excepto Belice. En Suramérica no hay registros publicados de Lu. Iongipalpis en Guyana, Surinam, Ecuador, Perú o Chile.

Además, presenta polimorfismos entre poblaciones a nivel morfológico, fisiológico, isoenzimático y molecular (21). Hasta la fecha se considera que el complejo Lu. longipalpis está conformado por cinco

\footnotetext{
Correspondencia:

Lina María Carrillo, Sede de Investigación Universitaria, SIU, Universidad de Antioquia, Carrera 53 N 61-30, laboratorio 632, Medellín, Colombia

Teléfono: (574) 219 6502, 219 6625; fax: (574) 2196511

linacarrillo@gmail.com

Recibido: 13/06/12; aceptado:23/06/12
}

especies monofiléticas (22), diferenciadas por métodos isoenzimáticos y marcadores moleculares. De estas cinco especies, solo una ha sido tipificada formalmente por parámetros morfológicos convencionales como Lu. pseudolongipalpis (23).

En Colombia, la presencia de Lu. longipalpis se ha reportado en gran parte del territorio nacional $(24,25)$, en zonas de bosque seco tropical (bs-t) según la clasificación de Holdridge (26). Su distribución ocurre principalmente a lo largo del valle del río Magdalena, incluyendo los departamentos de Caldas, Santander y algunos de la Costa Caribe, como Córdoba, Sucre y La Guajira (14). En relación con la distribución altitudinal, esta especie se ha encontrado predominantemente entre los 0 y 1.000 msnm. Varios reportes recientes la ubican a una altura máxima de $1.100 \mathrm{msnm}$ en el departamento de Huila (27) y en un rango de 930 y 1.300 msnm en un foco de leishmaniasis visceral en Piedecuesta, Santander (28).

Esta especie se ha adaptado en nuestro país a diferentes zonas, tanto áridas, generalmente reportada en América, como húmedas tropicales, caracterizadas por abundante vegetación, alta temperatura y humedad relativa, como en el caso de la reserva natural de río Claro, Antioquia, donde hasta ahora no se han reportado casos de leishmaniasis visceral. Sin embargo, la presencia del vector indica un riesgo para la transmisión de la enfermedad $(13,17,29)$.

El estudio de la distribución de los vectores, su ecología, la posible incriminación en focos y el riesgo epidemiológico en una región, permite importantes medidas de control en el manejo de la leishmaniasis. Los resultados que se presentan en este artículo se enmarcan en el trabajo de investigación de vigilancia entomología en el área de influencia de la hidroeléctrica Miel I.

En este artículo se informa por primera vez el hallazgo de Lu. longipalpis en los municipios de Marquetalia y Norcasia, en el departamento Caldas, y se reporta una nueva distribución altitudinal de dicha especie.

\section{Materiales y métodos \\ Área de estudio}

La recolección de los individuos se desarrolló en junio de 2007 en los municipios Victoria, La Dorada, Samaná, Norcasia y Marquetalia, en el departamento de Caldas, zona correspondiente al flanco oriental de la Cordillera Central de los 
Andes en Colombia (figura 1). Norcasia $\left(5^{\circ} 20^{\prime} \mathrm{N}\right.$ $\left.74^{\circ} 31^{\prime} O\right)$ tiene una altura promedio de 335 msnm, temperatura de $25{ }^{\circ} \mathrm{C}$ y pluviosidad de $4.340 \mathrm{~mm}$ anuales. Marquetalia $\left(15^{\circ} 18^{\prime} \mathrm{N}-75^{\circ} 3^{\prime} \mathrm{O}\right)$ presenta en promedio una altura de $1.600 \mathrm{msnm}$, temperatura de $20^{\circ} \mathrm{C}$ y pluviosidad de $3.126,4 \mathrm{~mm}$ anuales. Norcasia pertenece predominantemente al bosque húmedo tropical (bh-t) y, Marquetalia, al muy húmedo premontano (bmh-PM), según la clasificación de Holdridge (26).

\section{Método de captura}

El muestreo entomológico se hizo con trampas adhesivas impregnadas en aceite de ricino (30) dispuestas durante 10 días continuos, en dos épocas del año, de lluvia y de sequía. La intersección comprendió una distancia de 400 km, aproximadamente, divididos en 53 estaciones con 20 trampas en promedio por estación, las cuales fueron georreferenciadas. Los flebotomíneos se retiraron cuidadosamente y se transportaron al
Laboratorio del Programa de Estudio y Control de Enfermedades Tropicales (PECET) para su tratamiento e identificación. Posteriormente, se aclararon en lactofenol y se montaron sobre láminas portaobjeto, usando el medio de Hoyer. Para la identificación de especies, se usó la clave taxonómica de Young y Duncan, 1994.

\section{Resultados}

Se colocaron 2.34 trampas adhesivas y se capturaron 134 individuos pertenecientes a 14 especies del genero Lutzomyia. En el presente reporte se destaca la captura de cuatro individuos identificados como Lu. longipalpis, todos en época seca. Esta especie se recolectó en el municipio de Norcasia (un macho), en bosque muy húmedo tropical (bmh-T) a una altura de $392 \mathrm{msnm}$, y en Marquetalia (2 machos, 1 hembra), en bosque muy húmedo premontano (bmh-PM) a 1.387 msnm. Los ejemplares capturados en Marquetalia se almacenaron en la Colección de Vectores y

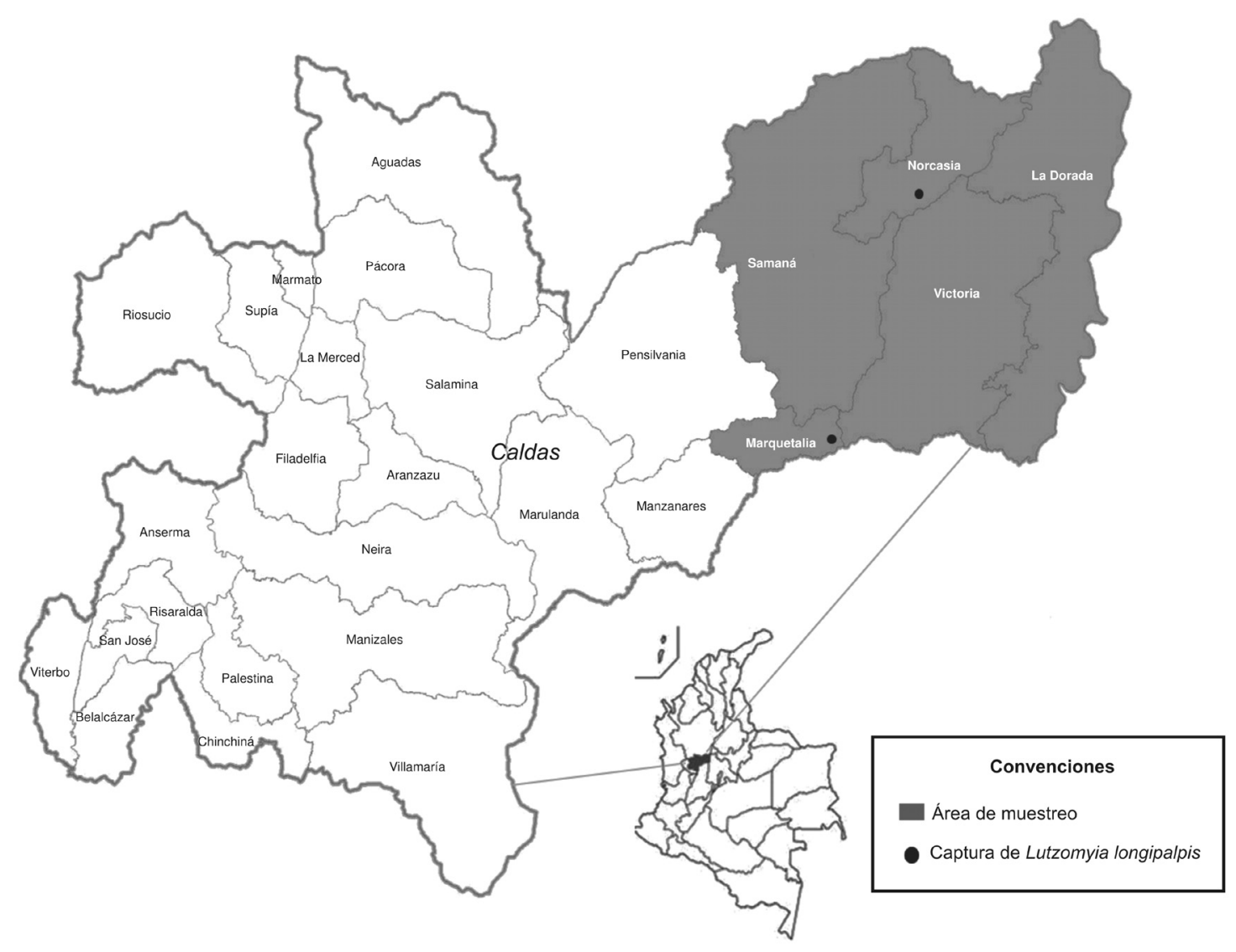

Figura 1. Área de estudio en el departamento de Caldas, que incluye los municipios de Norcasia, La Dorada, Victoria, Marquetalia y Samaná 
Hospedadores Intermediarios de Enfermedades Tropicales (VHET) del Programa de Estudio y Control de Enfermedades Tropicales. Códigos de acceso: PPCO3848 (đ) y PPCO 3852().

\section{Discusión}

El presente hallazgo constituye el primer registro de Lu. longipalpis en dos municipios del departamento de Caldas. Asimismo, se registra una altura máxima de distribución para esta especie nunca antes reportada en el país, correspondiente a 1.387 msnm en Marquetalia.

Aunque hasta la fecha no se han registrado casos de leishmaniasis visceral en ninguno de los dos municipios, la presencia de una especie antropofílica como Lu. longipalpis, tiene trascendencia epidemiológica, teniendo en cuenta el papel del vector como un focalizador de la enfermedad. La presencia de esta especie en dos nuevas localidades y a una altura máxima previamente sin reporte, se puede explicar por medio de dos hipótesis planteadas a continuación.

Como se describió anteriormente, desde hace aproximadamente 40 años se ha sugerido que $L u$. longipalpis es un complejo de especies. En 1969, Mangabeira (31), pionero en esta hipótesis, demostró que existían diferencias morfológicas entre individuos machos recolectados en varios estados de Brasil con diferentes condiciones ecológicas, y sugirió que se trataba de diferentes especies 0 variedades de esta. Desde entonces, varios autores han aplicado metodologías moleculares y bioquímicas para estudiar el fenómeno de especiación dentro del complejo Lu. longipalpis $(3,16,17,32)$.

Haffer, en 1969 (33), propuso que los periodos climáticos de lluvia y sequía durante el pleistoceno y en el periodo posterior al pleistoceno fueron determinantes para la formación de parches de bosques (teoría del refugio), e influyeron en la especiación geográfica y evolutiva de la fauna de América. Este patrón de distribución geográfica discontinua que presenta Lu. longipalpis a lo largo del neotrópico, sugiere que inicialmente pudo haber ocupado un área más amplia y, debido a cambios climáticos, presentó una distribución discontinua y sufrió un proceso de especiación por el bajo flujo genético $(32,34)$. Igualmente, los cambios ecológicos y demográficos en el continente americano, a lo largo de los años, pudieron dar lugar a condiciones propicias para la presencia y colonización de esta especie en regiones donde nunca había sido reportada previamente $(35,36)$.
Las secuelas del calentamiento global se han demostrado a nivel mundial; un claro ejemplo es el aumento observado de la isoterma de $0{ }^{\circ} \mathrm{C}$ en las montañas tropicales, aproximadamente, de $150 \mathrm{~m}$ desde el año 1970 (37). Los datos obtenidos de la medición de la temperatura diaria en la hidroeléctrica Miel I ubicada en Norcasia y facilitados por ISAGEN (no se presentan los datos), muestran que del 2003 al 2005 la temperatura aumentó $0,38^{\circ} \mathrm{C}$. Sin embargo, se necesitan más estudios y mediciones más precisas que abarquen periodos más extensos, para determinar el efecto del cambio climático en la zona.

Los insectos vectores son extremadamente sensibles al clima, tanto en su reproducción como en las tasas de mortalidad. Es posible formular la hipótesis que estos podrán ampliar sus hábitats a las regiones de mayor altura (37), ya que aquellos ecosistemas que son actualmente muy fríos para albergar estos vectores, se convertirán en otros más propicios para su existencia (38). Debido a que la reproducción de los vectores está influenciada por parámetros meteorológicos, es natural pensar que nuevos brotes de la enfermedad estén ligados al cambio climático y asumir una relación entre el aumento en la incidencia de la enfermedad y el calentamiento global (38).

En este estudio, el hallazgo de Lu. longipalpis en Norcasia y Marquetalia indica su posible adaptación a nuevos ambientes, pues hasta hace diez años, en un estudio de nuestro grupo que utilizó una metodología similar en esta mismazona, esta especie sólo fue recolectada en el municipio de La Dorada (39). Asimismo, en los registros entomológicos de los últimos diez años de la Secretaría de Salud de Caldas, no se reporta esta especie en los municipios descritos (comunicación personal).

Estos municipios se han venido transformando tanto estructural como tecnológicamente en conjunto con todo el país, especialmente por la construcción de la hidroeléctrica Miel I, lo que implica aumento de los asentamientos humanos, construcción de carreteras y mejoramiento de viviendas, entre otros aspectos (40). La expansión de las poblaciones humanas demanda una explotación cada vez mayor de los recursos naturales, lo que conlleva cambios en el tipo y la abundancia de la vegetación; esto crea, por lo tanto, diferentes nichos ecológicos y condiciones para la proliferación de vectores existentes que se adaptan u otros que recién llegan a la zona y que se adaptan a estas alteraciones (41). 
En varios estudios se ha demostrado que la migración humana debida a la sequía, a la degradación ambiental y a ciertos factores económicos, puede ampliar la distribución de las enfermedades de manera inesperada, y nuevos sitios de cría de vectores surgen debido a cambios climáticos y ambientales como la deforestación. El cambio climático exacerba estos efectos (42). Un claro ejemplo fue lo sucedido en Ceará, Brasil, con el aumento de casos de leishmaniasis visceral, en donde después de un periodo largo de sequía, tanto las poblaciones de humanos como de vectores se concentraron en zonas húmedas, alrededor de las fuentes de agua, lo que facilitó el contacto humanovector asociado a los cambios ambientales (5).

El aumento en la distribución de Lu. longipalpis demostrado en este estudio, puede deberse a varios factores como los que hemos descrito; estos, a su vez, no son excluyentes, pues es posible que el hecho de ser un complejo de especies, con amplia variabilidad genética entre poblaciones, favorezca su adaptabilidad a cambios ambientales como los del calentamiento global y facilite la ampliación de su distribución geográfica. En investigaciones previas de Barreto, et al. (27), y Flórez, et al. (28), se demostró la presencia de este vector en regiones de bosque seco tropical (bs-t) con una temperatura media de $24{ }^{\circ} \mathrm{C}$ en zonas con elevaciones entre los 930 y 1.300 msnm, que corresponden a las zonas de vida donde se presentan los casos de leishmaniasis visceral en Colombia. Sin embargo, su distribución no está limitada a este tipo de bosque; López (13) registró en el municipio de San Luis, Antioquia, la presencia de esta especie a una altura de 500 msnm en bosque húmedo tropical. Estos trabajos demuestran la plasticidad ecológica de Lu. longipalpis para sobrevivir en diversas condiciones climáticas, lo cual puede facilitar su establecimiento en zonas donde nunca antes se había registrado.

Estos hallazgos permiten suponer un potencial riesgo para la presencia de casos de leishmaniasis visceral en los municipios de Marquetalia y Norcasia, toda vez que existen las condiciones ecológicas y sociopolíticas, como la migración y desplazamiento forzado de personas no inmunes y animales infectados, que favorecerían la aparición de nuevas epidemias de la enfermedad (43-45).

Finalmente, debe declararse la alerta amarilla de riesgo para leishmaniasis visceral en los municipios de Marquetalia y Norcasia, pues, aunque fue un estudio puntual y la densidad de los vectores recolectados fue baja y ello es importante en la cuantificación de la alarma, no se debe desconocer la capacidad de Lu. longipalpis para adaptarse al peridomicilio en ambientes rurales y urbanos y el riesgo potencial que significa este registro en la zona de estudio. Esta transmisión depende de otros factores, como la presencia del parásito en reservorios como los perros y, población fluctuante, que debe ser vigilada en forma constante para evitar la presencia de casos en humanos (45). Por lo tanto, se vuelve necesario crear e implementar programas de vigilancia entomológica que puedan proveer información actualizada sobre la dinámica de la población de los vectores, ya que su adaptabilidad es creciente y de esto dependerá su control.

\section{Agradecimientos}

Agradecemos a las comunidades del oriente del departamento de Caldas y al personal de ISAGEN en la Hidroeléctrica Miel I.

\section{Conflicto de intereses}

Los autores no tienen ningún conflicto de intereses.

\section{Financiación}

Este trabajo se realizó dentro del marco del convenio 46/2160 entre ISAGEN y el Programa de Estudio y Control de Enfermedades Tropicales (PECET) de la Universidad de Antioquia.

\section{Referencias}

1. WHO. Control of the leishmaniases: Report of a meeting of the WHO Expert Committee on the Control of Leishmaniases. Geneva: WHO; 2010.

2. Bejarano EE, Uribe S, Rojas W, Vélez ID. Phlebotomine sand flies (Diptera: Psychodidae) associated with the appearance of urban leishmaniasis in the city of Sincelejo, Colombia. Mem Inst Oswaldo Cruz. 2002;97:645-7. http:// dx.doi.org/10.1590/S0074-02762002000500010

3. Soares RP, Turco SJ. Lutzomyia longipalpis (Diptera: Psychodidae: Phlebotominae): A review. An Acad Bras Cienc.2003;75:301-30.http://dx.doi.org/10.1590/S000137652003000300005

4. Lainson R, Rangel E. Lutzomyia longipalpis and the ecoepidemiology of American visceral leishmaniasis, with particular reference to Brazil -a review. Mem Inst Oswaldo Cruz.2005;100:811-27.http://dx.doi.org/10.1590/S007402762005000800001

5. Gage KL, Burkot TR, Eisen RJ, Hayes EB. Climate and vector borne diseases. Am J Prev Med. 2008;35:436-50. http://dx.doi.org/10.1016/j.amepre.2008.08.030

6. Organización Panamericana de la Salud. Informe final de la consulta de expertos OPS/OMS sobre leishmaniasis visceral en las Américas. Río de Janeiro, PANAFTOSA; 2006. 
7. Cárdenas R, Sandoval CM, Rodríguez AJ, Hernández D, Jaimes E, Mendoza JG. Medio ambiente y protozoosis sistémicas. II. Características fisiográficas del entorno y su asociación en la leishmaniasis visceral. Academia. 2004;3:35-40.

8. Anyamba A, Linthicum KJ, Small JL, Collins KM, Tucker CJ, Pak EW, et al. Climate teleconnections and recent patterns of human and animal disease outbreaks. PLoS Negl Trop Dis. 2012;6:e1465. http://dx.doi.org/10.1371/ journal.pntd.0001465

9. Lainson R, Ward RD, Shaw JJ. Experimental transmission of Leishmania chagasi, causative agent of neotropical visceral leishmaniasis, by the sandfly Lutzomyia longipalpis. Nature. 1977;266:628-30. http://dx.doi.org/10.1038/266628a0

10. Ramos-Santos C, Hernández-Montes $\mathbf{O}$, SánchezTejeda G, Monroy-Ostria A. Visceral leishmaniasis caused by Leishmania (L.) mexicana in a Mexican patient with human immunodeficiency virus infection. Mem Inst Oswaldo Cruz. 2000;95:729-33. http://dx.doi.org/10.1590/ S0074-02762000000500022

11. Montoya-Lerma J, Cadena H, Oviedo M, Ready PD, Barazarte R, Travi BL, et al. Comparative vectorial efficiency of Lutzomyia evansi and $L$. longipalpis for transmitting Leishmania chagasi. Acta Trop. 2003;85:19-29. http://dx.doi.org/10.1016/S0001-706X(02)00189-4

12. Young DG, Duncan MA. Guide to the identification and geographic distribution of Lutzomyia sand flies in Mexico, the West Indies, Central and South America (Diptera: Psychodidae). Mem Am Entomol Inst. 1994;54:1-881.

13. López Y, Osorio L, Álvarez G, Rojas J, Jiménez F, Gómez C, et al. Sandfly Lutzomyia longipalpis in a cutaneous leishmaniasis focus in central Colombia. Mem Inst Oswaldo Cruz.1996;91:415-9.http://dx.doi.org/10.1590/S007402761996000400005

14. González C, Cabrera OL, Munstermann LE, Ferro C. Distribución de los vectores de Leishmania infantum (Kinetoplastida: Trypanosomatidae) en Colombia. Biomédica. 2006;26:64-72.

15. Ward RD, Ribeiro AL, Ready PR, Murtagh A. Reproductive isolation between different forms of Lutzomyia longipalpis (Lutz \& Neiva), (Diptera: Psychodidae), the vector of Leishmania donovani chagasi Cunha \& Chagas and its significance to kala azar distribution in South America. Mem Inst Oswaldo Cruz. 1983;78:269-80.

16. Uribe S. Polimorfismo entre poblaciones de Lutzomyia longipalpis, insecto transmisor de leishmaniasis visceral, sugiere la existencia de un complejo de especies. Biomédica. 1999;19:56-66.

17. Uribe S. The status of the Lutzomyia longipalpis species complex and possible implications for leishmania transmission. Mem Inst Oswaldo Cruz. 1999;94:729-34. http://dx.doi.org/10.1590/S0074-02761999000600005

18. Arrivillaga JC, Feliciangeli MD. Diferenciación morfológica larval entre poblaciones de Lutzomyia longipalpis (Lutz \& Neiva 1912) en Venezuela (Diptera: Psychodidae). Bol Entomol Venez. 2000;15:229-34.

19. Arrivillaga J, Salerno P, Rangel Y. Aislamiento reproductivo asimétrico entre Lutzomyia pseudolongipalpis y Lutzomyia longipalpis (especie C2), vectores neotropicales de leishmaniasis visceral (Diptera: Psychodidae). Rev Biol Trop. 2009;57:23-31.

20. Grimaldi G, Tesh RB, McMahon-Pratt D. A review on the geographic distribution and epidemiology of Leishmaniasis in the New World. Am J Trop Med Hyg. 1989;41:687-725.

21. Azevedo A, Monteiro F, Cabello P, de Souza N, Goreti M, Rangel E. Studies on populations of Lutzomyia longipalpis (Lutz \& Neiva, 1912) (Diptera: Psychodidae: Phlebotominae) in Brazil. Mem Inst Oswaldo Cruz. 2000;95:305-22. http:// dx.doi.org/10.1590/S0074-02762000000300005

22. Arrivillaga JC, Mutebi JP, Piñango H, Norris D, Alexander $\mathrm{B}$, Feliciangeli MD, et al. The taxonomic status of genetically divergent populations of Lutzomyia longipalpis (Diptera: Psychodidae) based on the distribution of mitochondrial and isozyme variation. J Med Entomol. 2003;40:615-27.

23. Arrivillaga JC, Feliciangeli MD. Lutzomyia pseudolongipalpis: The first new species within the Longipalpis (Diptera: Psychodidae: Phlebotominae) complex from La Rinconada, Curarigua, Lara State, Venezuela. J Med Entomol. 2001;38:783-90.

24. Montoya-Lerma J, Ferro C. Flebótomos (Diptera: Psychodidae) de Colombia. En: Amat G, Andrade MG, Fernández $F$, editores. Insectos de Colombia. Colección Jorge Álvarez Lleras, No. 13, Academia Colombiana de Ciencias Exactas, Físicas y Naturales. Santafé de Bogotá: Centro Editorial Javeriano; 1999. p. 211-45.

25. Bejarano EE. Lista actualizada de los psicódidos (Diptera: Psychodidae) de Colombia. Folia Entomol Mex. 2006;45:47-56.

26. Holdridge LR. Ecología basada en zonas de Vida. $1^{\text {a }}$ edición. San José de Costa Rica: Editorial IICA; 1979.

27. Barreto M, Burbano ME, Barreto P. Registros de Lutzomyia (Diptera: Psychodidae) en nuevas localidades de Colombia. Colomb Med. 2006;37:39-45.

28. Flórez M, Martínez JP, Gutiérrez R, Luna KP, Serrano VH, Ferro C, et al. Lutzomyia longipalpis (Diptera: Psychodidae) en un foco suburbano de leishmaniosis visceral en el Cañón del Chicamocha en Santander, Colombia. Biomédica. 2006;26(Supl.1):109-20.

29. Vivero RJ, Muskus CE, Uribe SI, Bejarano EE, Torres C. Flebotomíneos (Diptera: Psychodidae) en la Reserva Natural del Cañón del Río Claro (Antioquia), Colombia. Actual Biol. 2010;32:165-71.

30. Maroli M, Feliciangeli MD, Arias J. Métodos de captura, conservación y montaje de los flebótomos (Diptera: Psychodidae). Washington, D.C.: OPS; 1997. p. 1-67.

31. Mangabeira 0 . Sobre a sistemática e biologia dos Phlebotomus do Ceará. Rev Bras Malariol Doenças Trop. 1969;21:3-26.

32. Bauzer LG, Souza NA, Maingon RD, Peixoto AA. Lutzomyia longipalpis in Brazil: a complex or a single species? A minireview. Mem Inst Oswaldo Cruz. 2007;102:1-12. http:// dx.doi.org/10.1590/S0074-02762007000100001

33. Haffer J. Speciation in Amazonian forest birds. Science. 1969;165:131-7.http://dx.doi.org/10.1126/science.165. 3889.131

34. Arrivillaga JC, Norris DE, Feliciangeli MD, Lanzaro GC. Phylogeography of the neotropical sand fly Lutzomyia 
longipalpis inferred from mitochondrial DNA sequences Infect Genet Evol. 2002;2:83-95. http://dx.doi.org/10.1016/ S1567-1348(02)00087-4

35. Lainson R. Demographic changes and their influence on the epidemiology of the American leishmaniases. In: Service MW, editor. Demography and vetor-borne disease. Boca Raton, Florida: CRC Press, Inc.; 1988. p. 85-106.

36. Tesh RB. Control of zoonotic visceral leishmaniasis: Is it time to change strategies? Am J Trop Med Hyg. 1995;52:287-92.

37. Zeli R. Global climate change and the emergence/reemergence of infectious diseases. Int $\mathrm{J}$ Med Microbiol. 2004;293:16-26. http://dx.doi.org/10.1016/S1433-1128(04) 80005-6

38. Khasnis AA, Nettleman MD. Global warming and infectious disease. Arch Med Res. 2005;36:689-96. http://dx.doi. org/10.1056/NEJMp0912931

39. Vélez ID, Agudelo LA, Cuartas SE, Arango M, Díaz MR. Determinación del riesgo de infección por Leishmania spp. en la zona de influencia del Proyecto Hidroeléctrico Miel I en el departamento de Caldas. Rev ECM. 1999;4:45-59.

40. Alcaldía de Marquetalia. Datos sobre Marquetalia Fecha de consulta: 20 de octubre de 2007. Disponible en: http://www.marquetalia-caldas.gov.co/nuestromunicipio. shtml?apc=l-xx-1-\&s=m\&m=I.
41. Patz JA, Graczyk TK, Geller N, Vittor AY. Effects of environmental change on emerging parasitic diseases. Int J Parasitol. 2000;30:1395-405. http://dx.doi.org/10.1016/ S0020-7519(00)00141-7

42. Githeko AK, Lindsay SW, Confalonieri UE, Patz JA. Climate change and vector-borne diseases: A regional analysis. Bull World Health Organ. 2000;78:1136-47.

43. OPS/OMS. Tendencias demográficas y de mortalidad en la región de las Américas, 1980-2000. Boletín Epidemiológico. 2002;23:1-16.

44. Rebollar-Téllez E, Ramírez-Fraire A, Andrade-Narváez F. A two years study on vector of cutaneous leishmaniasis. Evidence for sylvatic transmission cycle in the State of Campeche, México. Mem Inst Oswaldo Cruz. 1996;91:55560. http://dx.doi.org/10.1590/S0074-02761996000500004

45. Sandoval C, Angulo V, Gutiérrez R, Muñoz G, Ferro C. Especies de Lutzomyia (Diptera: Psychodidae) posibles vectores de leishmaniasis en la ciudad de Bucaramanga, Santander, Colombia. Biomédica. 1998;18:161-8.

46. Dantas-Torres F. The role of dogs as reservoirs of Leishmania parasites, with emphasis on Leishmania (Leishmania) infantumand Leishmania(Viannia) braziliensis. Vet Parasitol. 2007;149:139-46. http://dx.doi.org/10.1016/j. vetpar.2007.07.007 